\title{
Optimal Energy-Efficient Sensing and Power Allocation in Cognitive Radio Networks
}

\author{
Xia Wu, ${ }^{1,2}$ Jin-Ling Xu, ${ }^{2}$ Ming Chen, ${ }^{2}$ and Junbo Wang ${ }^{2}$ \\ ${ }^{1}$ Department of Mathematics, Southeast University, Nanjing 210000, China \\ ${ }^{2}$ National Mobile Communications Research Laboratory, Southeast University, Nanjing 210000, China \\ Correspondence should be addressed to Xia Wu; wuxia80@seu.edu.cn
}

Received 29 April 2014; Accepted 21 July 2014; Published 5 August 2014

Academic Editor: Mohamed Djemai

Copyright (c) 2014 Xia Wu et al. This is an open access article distributed under the Creative Commons Attribution License, which permits unrestricted use, distribution, and reproduction in any medium, provided the original work is properly cited.

\begin{abstract}
We consider a joint optimization of sensing parameter and power allocation for an energy-efficient cognitive radio network (CRN) in which the primary user $(\mathrm{PU})$ is protected. The optimization problem to maximize the energy efficiency of CRN is formulated as a function of two variables, which are sensing time and transmit power, subject to the average interference power to the PU and the target detection probability. During the optimizing process, the quality of service parameter (the minimum rate acceptable to secondary users (SUs)) has also been taken into consideration. The optimal solutions are analyzed and an algorithm combined with fractional programming that maximizes the energy efficiency for CRN is presented. Numerical results show that the performance improvement is achieved by the joint optimization of sensing time and power allocation.
\end{abstract}

\section{Introduction}

The traditional approach of fixed spectrum allocation leads to spectrum underutilization with the fast development of wireless communications. It is reported that there are vast temporal and spatial variations in the usage of allocated spectrum, which can be as low as $15 \%$ in recent studies by the FCC $[1,2]$. This motivates the concept of CRN that allows SUs to opportunistically exploit the underutilized spectrum. In other words, CRN enables SUs to share the spectrum bands opportunistically with PUs.

In a practical CRN which is powered by batteries, the concept of energy-efficient design is essential. Both the sensing time and the transmission power affect the energy efficiency of a CRN. For example, if the sensing time is too short, it may reduce the energy consumption in sensing phase and increase the transmission time, but data loss probability and interference to the PU are also introduced due to false detection. If the sensing time swells, detection accuracy is improved. However, it consumes more energy without ameliorating data transmission. Similarly, transmission power is also salient from the viewpoint of energy efficiency. The larger the transmission power is, the more throughput can be obtained. However, it consumes more energy.
Some recent works dealing with the issues of CRN's energy efficiency have been presented in [3-7]. In [3], the authors discuss the CRN's energy-efficient transmission with variable transmission duration and interference to the PU when the sensing duration is fixed. In [4], a joint design of energy-efficient sensing and transmission durations for a CRN is considered. In [5], a joint optimization problem of the fusion rule threshold and detector's threshold to maximize the energy efficiency of the CRN is studied. In [6], the authors identify the sensing-access strategies and the sensing order that achieve the maximum energy efficiency. In [7], the authors propose a low complexity solution to the problem of finding the power allocation that maximizes the energy efficiency, while limiting the interference to the PU and meeting the SUs' minimum rate requirements.

In this paper, we will focus on the joint optimization between the spectrum sensing time and power allocation to maximize energy efficiency, which is defined as the number of transmitted data bits per Joule of energy, with limited interference power to the PU. During the optimizing process, the target detection probability and the quality of service parameter (such as the minimum rate acceptable to SU) have also been taken into consideration. We consider the scenario that multiple SUs jointly sense one licensed spectrum band 
which can be divided into multiple subbands, and each SU operates exclusively in one of the subbands when the PU is detected to be absent. An optimization algorithm combined with fractional programming to solve the optimization problem is proposed. We compare the proposed scheme that jointly optimizes sensing time and power allocation with the schemes that only optimize either sensing time or power allocation, and numerical results demonstrate that the performance improvement is achieved by the joint optimization of sensing time and power allocation.

The rest of this paper is organized as follows. In Section 2, we present the system model. In Section 3, we address an optimization problem for an energy-efficient CRN. In Section 4, the optimization problem is analyzed and an algorithm combined with fractional programming is given to achieve the optimum value. Then we present numerical results in Section 5. Finally, we conclude our paper in Section 6.

\section{System Model}

2.1. System Model. In this paper we consider a CRN where multiple SUs are allowed to access one licensed spectrum band. The entire licensed band is divided into $M$ subbands, and each SU operates exclusively in one of the subbands when the PU is detected to be absent. Such assumption is widely used in $[7,8]$.

The system is assumed to be perfectly synchronized, and time is divided into slots, each with a fixed length $T$. Each slot includes three phases [9]: (1) the sensing phase, (2) the reporting phase, and (3) the transmission phase. We assume that the PU is either absent or present during each slot. During the sensing time, the SUs need to sense the PU's activity. Since the PU will operate in all the subbands once becoming active, the SUs can jointly sense the PU's presence and send the spectrum results to the fusion center via a common control channel [5] in the reporting phase. Finally, based on the spectrum sensing results of the SUs, the fusion center determines the absent or active status of PU. With the cooperative spectrum sensing, each SU can spend less time detecting while enjoying a low false alarm probability. In addition, to keep the duration of the reporting phase at an acceptable level, the number of SUs that are involved should be limited. It can be seen that the reporting phase adds a fixed time overhead [10]. Without loss of generality, the duration of the reporting phase is assumed to be zero. The transmission phase is used for data transmission of SUs when the PU is detected to be absent. The structure of the time slot is shown in Figure 1, where $\tau_{s}$ denotes the sensing time, $\tau_{r}$ represents the reporting time of single $\mathrm{SU}$, and $T_{t}$ is the transmission time.

2.2. Cooperative Spectrum Sensing. The spectrum sensing is considered as a binary hypothesis testing problem: hypothesis $H_{0}$ when the PU is absent and hypothesis $H_{1}$ when the PU is active. For the energy detection scheme, the test statistic for the energy detector of the $i$ th SU is compared with a detection threshold $\varepsilon$, to decide the absent or active status of PU. Denote $\gamma_{i}$ is the received average SNR of PU's signal measured at the

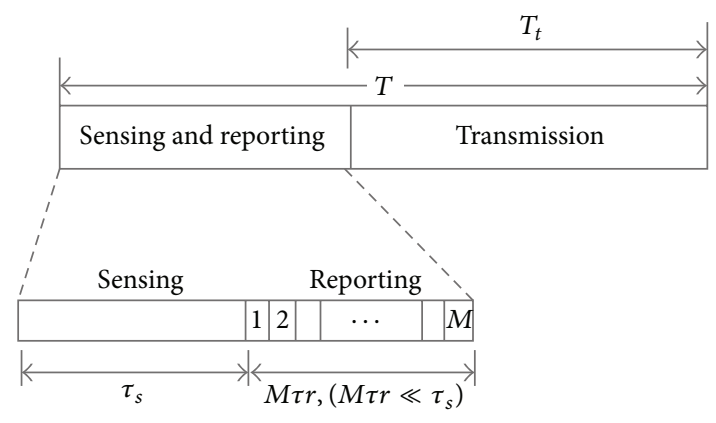

FIGURE 1: Structure of the time slot.

$i$ th secondary detector under hypothesis $H_{1}$. Under AWGN fading channel assumption, the probabilities of detection and false alarm of the $i$ th SU are given, respectively, by [11]

$$
\begin{aligned}
& P_{d}\left(\tau_{s}, \varepsilon\right)=Q\left(\frac{\varepsilon-\tau_{s} f_{s}-\gamma_{i}}{\sqrt{2 \tau_{s} f_{s}+4 \gamma_{i}}}\right), \\
& P_{f}\left(\tau_{s}, \varepsilon\right)=Q\left(\frac{\varepsilon-\tau_{s} f_{s}}{\sqrt{2 \tau_{s} f_{s}}}\right),
\end{aligned}
$$

where $\tau_{s}$ represents the sensing time, $f_{s}$ denotes the sampling frequency, and finally the $Q(\cdot)$ is the complementary distribution function of standard Gaussian.

The "most rule" is used to make the final decision by the fusion center, where at least half of SUs' decisions say that the $\mathrm{PU}$ is active; then the final decision declares that the PU is active [11]. Note that our model can be readily extended to different cooperative spectrum sensing schemes. Similar to [12], we assume that the size of the CRN is small compared with its distance from the primary system. Therefore, the received signal at each $\mathrm{SU}$ experiences almost identical path loss. Hence each SU has the same $P_{d}$ and $P_{f}$. For a given time slot, $M$ SUs perform spectrum sensing; the final probabilities of false alarm and detection based on the most rule are given, respectively, by [12]

$$
\begin{aligned}
& Q_{F}\left(\tau_{s}, \varepsilon\right)=\sum_{m=\lceil M / 2\rceil}^{M} C_{M}^{m} P_{f}\left(\tau_{s}, \varepsilon\right)^{m}\left(1-P_{f}\left(\tau_{s}, \varepsilon\right)\right)^{M-m}, \\
& Q_{D}\left(\tau_{s}, \varepsilon\right)=\sum_{m=\lceil M / 2\rceil}^{M} C_{M}^{m} P_{d}\left(\tau_{s}, \varepsilon\right)^{m}\left(1-P_{d}\left(\tau_{s}, \varepsilon\right)\right)^{M-m}
\end{aligned}
$$

where $C_{M}^{m}=M ! / m !(M-m) !$.

\section{Problem Formulation}

3.1. Average Throughput of CRN. The spectrum band's availability for SUs' transmission is based on the outcome of the spectrum sensing. If the $\mathrm{PU}$ is detected to be absent while the PU is actually active, the PU's signal is an interference to SUs' transmission, and we assume the transmission will not succeed. Hence, when the PU is absent and no false alarm is generated, the SUs can successfully transmit the data. 
Denote $C_{i}$ as the achievable data rate when the $i$ th SU transmits under no interference from the PU over the subband labeled $i$, and the value of $C_{i}$ is assumed to be [11]

$$
C_{i}=\log _{2}\left(1+\frac{g_{i} p_{i}}{N_{0}}\right)
$$

where $p_{i}$ represents the transmission power of the $i$ th SU, $g_{i}$ denotes the gain on subband $i$, and $N_{0}$ is the background noise power per $\mathrm{Hz}$.

The average throughput in a time slot $T$ on subband $i$ can be expressed as

$$
R_{i}\left(\tau_{s}, p_{i}, \varepsilon\right)=\left(T-\tau_{s}\right) P_{0}\left(1-Q_{F}\left(\tau_{s}, \varepsilon\right)\right) C_{i},
$$

where $P_{0}$ denotes the absent probability of the PU.

Therefore, the average throughput of the CRN in a time $\operatorname{slot} T$ is

$$
R\left(\tau_{s}, \mathbf{p}, \varepsilon\right)=\sum_{i=1}^{M} R_{i}\left(\tau_{s}, p_{i}, \varepsilon\right) .
$$

If $r_{i}$ is the minimum rate acceptable to the $i$ th SU, then we have

$$
\log _{2}\left(1+\frac{g_{i} p_{i}}{N_{0}}\right) \geq r_{i}, \quad \text { for } i=1, \ldots, M .
$$

If we let $\left(2^{r_{i}}-1\right) N_{0} / g_{i}=\bar{P}_{i}$, we can rewrite (7) as

$$
p_{i} \geq \bar{P}_{i}, \quad \text { for } i=1, \ldots, M .
$$

It is easy to observe that the SUs will cause interference to the $\mathrm{PU}$ when $\mathrm{PU}$ is active. Therefore, the average interference power constraint can be written as [13]

$$
\sum_{i=1}^{M} P_{1}\left(1-Q_{D}\left(\tau_{s}, \varepsilon\right)\right) g_{i} P_{i} \leq I_{\text {th }},
$$

where $I_{\text {th }}$ represents an acceptable average interference threshold of PU and $P_{1}=1-P_{0}$ denotes the active probability of the PU.

3.2. Average Energy Consumption of CRN. In our model, the energy consumption of the $i$ th SU consists of three parts: circuit consumption power $P_{c}$, spectrum sensing power $P_{s}$, and data transmit power $p_{i}$.

In the $\mathrm{CRN}$, there are four different scenarios between the activities of the PU and the SUs.

(S1) In this scenario, the SUs successfully detect the PU's absent status with probability $P_{0}\left(1-Q_{F}\left(\tau_{s}, \varepsilon\right)\right)$, and the energy consumed is given by

$$
C_{1}\left(\tau_{s}, \mathbf{p}\right)=M\left(P_{s}+P_{c}\right) \tau_{s}+\left(T-\tau_{s}\right)\left(\sum_{i=1}^{M} p_{i}+M P_{c}\right) \text {. }
$$

(S2) For this scenario, the SUs falsely detect the PU's absent status with probability $P_{0} Q_{F}\left(\tau_{s}, \varepsilon\right)$, and the energy used is given by

$$
C_{2}\left(\tau_{s}, \mathbf{p}\right)=M\left(P_{s}+P_{c}\right) \tau_{s} .
$$

(S3) In this case, the SUs falsely detect the PU's active status with probability $P_{1}\left(1-Q_{D}\left(\tau_{s}, \varepsilon\right)\right)$, and the energy used is given by

$$
C_{3}\left(\tau_{s}, \mathbf{p}\right)=C_{1}\left(\tau_{s}, \mathbf{p}\right) .
$$

(S4) Under this scenario, the SUs successfully detect the PU's active status with probability $P_{1} Q_{D}\left(\tau_{s}, \varepsilon\right)$, and the energy consumed is given by

$$
C_{4}\left(\tau_{s}, \mathbf{p}\right)=C_{2}\left(\tau_{s}, \mathbf{p}\right)
$$

The average energy consumption in a time slot $T$ can be expressed by

$$
\begin{aligned}
\psi\left(\tau_{s}, \mathbf{p}, \varepsilon\right)= & M P_{s} \tau_{s}+M P_{c} \tau_{s}+\left(T-\tau_{s}\right) \\
& \cdot\left(1-P_{0} Q_{F}\left(\tau_{s}, \varepsilon\right)-P_{1} Q_{D}\left(\tau_{s}, \varepsilon\right)\right) \\
& \cdot\left(M P_{c}+\sum_{i=1}^{M} p_{i}\right) .
\end{aligned}
$$

3.3. Energy Efficiency. Our objective is to maximize the energy efficiency of the CRN subject to the target detection probability constraint while keeping the average interference introduced to the PU below a certain threshold, which can be expressed as

$$
\begin{array}{ll}
\max _{\tau_{s}, \mathbf{p}, \varepsilon} & \eta\left(\tau_{s}, \mathbf{p}, \varepsilon\right)=\frac{R\left(\tau_{s}, p_{i}, \varepsilon\right)}{\psi\left(\tau_{s}, p_{i}, \varepsilon\right)} \\
\text { subject to } & 0<\tau_{s}<T \\
& Q_{D}\left(\tau_{s}, \varepsilon\right)=\alpha \\
& Q_{F}\left(\tau_{s}, \varepsilon\right) \leq \beta \\
& p_{i} \geq \bar{P}_{i} \\
& \sum_{i=1}^{M} P_{1}\left(1-Q_{D}\left(\tau_{s}, \varepsilon\right)\right) g_{i} p_{i} \leq I_{\text {th }},
\end{array}
$$

where $\alpha$ is the target detection probability.

Notably, the above problem is not convex, and directly obtaining the optimal solution is difficult. Hence, in the next section, fractional programming [14] is utilized to solve it.

\section{Solution of Formulation}

In this section, we will apply optimization algorithm combined with fractional programming to solve problem (15a)(15f).

When the constraint $(15 \mathrm{c})$ is considered only, for any given $\tau_{s}$, we can determine a threshold from (1) that is able to satisfy constraint $(15 \mathrm{c})$, which is given by

$$
\varepsilon\left(\tau_{s}\right)=\sqrt{2 \tau_{s} f_{s}+4 \gamma_{i}} Q^{-1}\left(\bar{P}_{d}\right)+\tau_{s} f_{s}+\gamma_{i},
$$

where $\bar{P}_{d}$ is the single SU detection probability satisfying $Q_{D}\left(\tau_{s}, \varepsilon\right)=\alpha$. 
Similarly, when the constraint (15d) is considered only, the optimal solution occurs only when constraint (15d) is at equality. The proof is similar to [12]. When constraint (15d) is at equality, for any given $\tau_{s}$, we can determine a threshold from (2) that is able to satisfy constraint (15d), which is given by

$$
\varepsilon\left(\tau_{s}\right)=\sqrt{2 \tau_{s} f_{s}} Q^{-1}\left(\bar{P}_{f}\right)+\tau_{s} f_{s}
$$

where $\bar{P}_{f}$ is the single SU detection probability satisfying $Q_{F}\left(\tau_{s}, \varepsilon\right)=\beta$.

In order to satisfy both constraints (15c) and (15d), $\varepsilon\left(\tau_{s}\right)$ should choose the max value between (16) and (17):

$$
\begin{aligned}
\varepsilon\left(\tau_{s}\right)=\max \{ & \sqrt{2 \tau_{s} f_{s}+4 \gamma_{i}} Q^{-1}\left(\bar{P}_{d}\right)+\tau_{s} f_{s} \\
& \left.+\gamma_{i}, \sqrt{2 \tau_{s} f_{s}} Q^{-1}\left(\bar{P}_{f}\right)+\tau_{s} f_{s}\right\} .
\end{aligned}
$$

Since constraints (15c) and (15d) can be satisfied by (18) for any given $\tau_{s}$, the optimization $(15 \mathrm{a})-(15 \mathrm{f})$ is reduced to

$$
\begin{array}{ll}
\max _{\tau_{s}, \mathbf{p}} & \eta\left(\tau_{s}, \mathbf{p}\right)=\frac{R\left(\tau_{s}, p_{i}, \varepsilon\left(\tau_{s}\right)\right)}{\psi\left(\tau_{s}, p_{i}, \varepsilon\left(\tau_{s}\right)\right)} \\
\text { subject to } & 0<\tau_{s}<T \\
& p_{i} \geq \bar{P}_{i} \\
& \sum_{i=1}^{M} P_{1} q_{m} g_{i} p_{i} \leq I_{\mathrm{th}},
\end{array}
$$

where $q_{m}=1-\alpha$.

Since $\tau_{s}$ lies within the limited interval $(0, T)$, similar to [15], we can exhaustively search for the optimal sensing time over $(0, T)$. Hence, for a given $\tau_{s}$, problem (19a)-(19d) can be simplified as follows:

$$
\begin{array}{ll}
\max _{\mathbf{p}} & \eta(\mathbf{p})=\frac{c_{1} \sum_{i=1}^{M} \log _{2}\left(1+g_{i} p_{i} / N_{0}\right)}{c_{2}+c_{3} \sum_{i=1}^{M} p_{i}} \\
\text { subject to } & p_{i} \geq \bar{P}_{i} \\
& \sum_{i=1}^{M} P_{1} q_{m} g_{i} p_{i} \leq I_{\mathrm{th}},
\end{array}
$$

where $c_{1}=\left(T-\tau_{s}\right) P_{0}\left(1-Q_{F}\left(\tau_{s}, \varepsilon\right)\right), c_{3}=\left(T-\tau_{s}\right)(1-$ $\left.P_{0} Q_{F}\left(\tau_{s}, \varepsilon\right)-P_{1} \alpha\right)$, and $c_{2}=M P_{s} \tau_{s}+M P_{c} \tau_{s}+c_{3} M P_{c}$.

The fractional programming [14] can be used to solve problem (20a)-(20c). First, a new objective function is defined as

$$
h(\mathbf{p}, \lambda)=c_{1} \sum_{i=1}^{M} \log _{2}\left(1+\frac{g_{i} p_{i}}{N_{0}}\right)-\lambda\left(c_{2}+c_{3} \sum_{i=1}^{M} p_{i}\right),
$$

where $\lambda$ is a positive parameter.
Hence, another problem is formulated as

$$
\begin{array}{ll}
\max _{\mathbf{p}} & h(\mathbf{p}, \lambda) \\
\text { subject to } & p_{i} \geq \bar{P}_{i} \\
& \sum_{i=1}^{M} P_{1} q_{m} g_{i} p_{i} \leq I_{\mathrm{th}} .
\end{array}
$$

Define the optimal value and solution of problem $(22 \mathrm{a})-$ (22c) as $F(\lambda)=\max _{\mathbf{p}}\{h(\mathbf{p}, \lambda)\}$ and $\widehat{\mathbf{p}}(\lambda)=\arg \max _{\mathbf{p}}\{h(\mathbf{p}, \lambda)\}$, respectively. The following lemma introduced by Dinkelbach [14] can relate problem (20a)-(20c) and (22a)-(22c), and detailed proof can be found in [14].

Lemma 1. The optimal solution $\mathbf{p}^{*}$ achieves the optimal value $\lambda^{*}$ of problem (20a)-(20c); that is, $\lambda^{*}=\max _{\mathbf{p}}\{\eta(\mathbf{p})\}=\eta\left(\mathbf{p}^{*}\right)$ according to (20a), if and only if

$$
F\left(\lambda^{*}\right)=0, \quad \widehat{\mathbf{p}}(\lambda)=\mathbf{p}^{*} .
$$

This lemma indicates that at the optimal parameter $\lambda^{*}$, the optimal solution to problem $(22 \mathrm{a})-(22 \mathrm{c})$ is also the optimal solution to problem (20a)-(20c). Hence, solving problem (20a)-(20c) can be realized by finding the optimal power allocation of problem (22a)-(22c) for a given $\lambda$ and then update $\lambda$ until (23) is established.

For a given $\lambda$, the optimal power allocation can be obtained using convex theory [16] because of the characteristic of problem (22a)-(22c). First, the constraint (22b) is not considered; then the Lagrange function of problem (22a)$(22 \mathrm{c})$ is

$$
\begin{aligned}
L= & c_{1} \sum_{i=1}^{M} \log _{2}\left(1+\frac{g_{i} p_{i}}{N_{0}}\right)-\lambda\left(c_{2}+c_{3} \sum_{i=1}^{M} p_{i}\right) \\
& -\mu\left(\sum_{i=1}^{M} P_{1} q_{m} g_{i} p_{i}-I_{\text {th }}\right),
\end{aligned}
$$

where $\mu$ is the Lagrange multiplier for constraint (22c). Using the Karush-Kuhn-Tucker (KKT) condition, the optimal solution for problem (22a)-(22c) without constraint (22b) is

$$
p_{i}=\max \left\{\frac{c_{1}}{\ln 2\left(\lambda c_{3}+\mu P_{1} q_{m} g_{i}\right)}-\frac{N_{0}}{g_{i}}, 0\right\}
$$

and the value of $\mu$ can be found by subgradient method [16].

The optimal value of problem (22a)-(22c) without constraint (22b) can be obtained by Algorithm 1 .

In the following, we classify the optimal value obtained by Algorithm 1 into two cases considering the constraint (22b); we start with Case 1, where $S$ represents the set of all SUs in the CRN.

Case 1. When $p_{i} \geq \bar{P}_{i}$ for all SUs, the maximum energy efficiency of problem (20a)-(20c) is $\eta(\mathbf{p})=\lambda^{*}$.

Case 2. $p_{i} \geq \bar{P}_{i}$ for $i \in S_{1}$, and $p_{i}<\bar{P}_{i}$ for $i \in S_{2}$. Note that for those $i \in S_{2}$ the only way to satisfy the condition (22b) is to set

$$
p_{i}=\bar{P}_{i}, \quad \text { for } i \in S_{2} \text {. }
$$




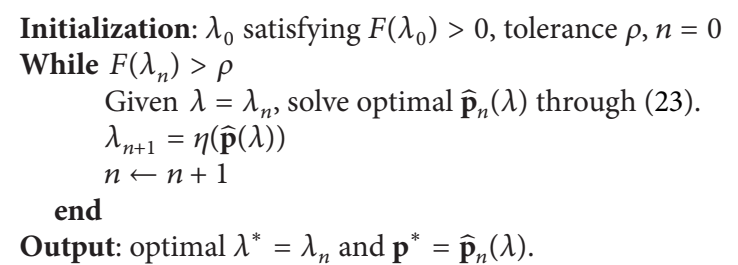

Algorithm 1: Fractional programming to solve problem (20a)-(20c) without the constraint (20b).

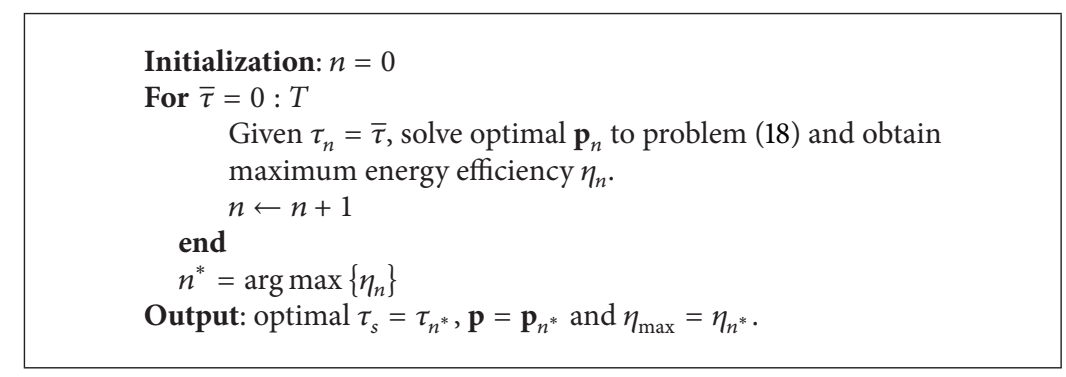

Algorithm 2: Optimal sensing time and power allocation for energy-efficient CRN.

After assigning these powers to SUs who fall in $S_{2}$, we can reformulate the problem (20a)-(20c) with optimization variables $p_{i}$ for $i \in S_{1}$ and fixed $p_{i}=\bar{P}_{i}$ for $i \in S_{2}$ and resolve it through the above method.

Hence, the optimal solution for problem (15a)-(15f) is given in Algorithm 2.

\section{Numerical Results}

The default parameters are set as follows: the number of SUs $M=8$, the fixed time slot $T=20 \mathrm{~ms}$, and the sampling frequency of the received signal is assumed to be $6 \mathrm{MHz}$. The SNR of the PU's signal received at the SUs is set to be $-20 \mathrm{~dB}$. The background noise power is $N_{0}=10^{-2} \mathrm{~W} / \mathrm{Hz}$. The active probability of the channel is set to be $P_{1}=0.3$. The threshold of the detection probability on all channel is assumed to be $\alpha=0.9$. The threshold of the false alarm probability on all channel is $\beta=0.2$. The sensing and circuit power are set as $0.02 \mathrm{~W}$ and $0.01 \mathrm{~W}$, respectively. The minimum rate requirement is $2 \mathrm{bit} / \mathrm{s} / \mathrm{Hz}$; we consider a Rayleigh fading environment with average channel power gain, that is, $E\left\{g_{i}\right\}$ equal to 0.1 .

In Figure 2, the energy efficiency versus the sensing time is compared among different SNR when power allocation is optimized with the fixed average interference power $I_{\text {th }}=$ $-20 \mathrm{dBW}$. It can be observed that when sensing time initially increases, the energy efficiency increases. This means that the improvement in sensing performance is able to outweigh the loss in shorter transmission time and larger energy used for sensing. However, when sensing time is increased further, the energy efficiency decreases as it is no longer worth to improve the sensing performance. Furthermore, when the SNR is high, the optimal sensing time is shorter and the energy efficiency is larger.

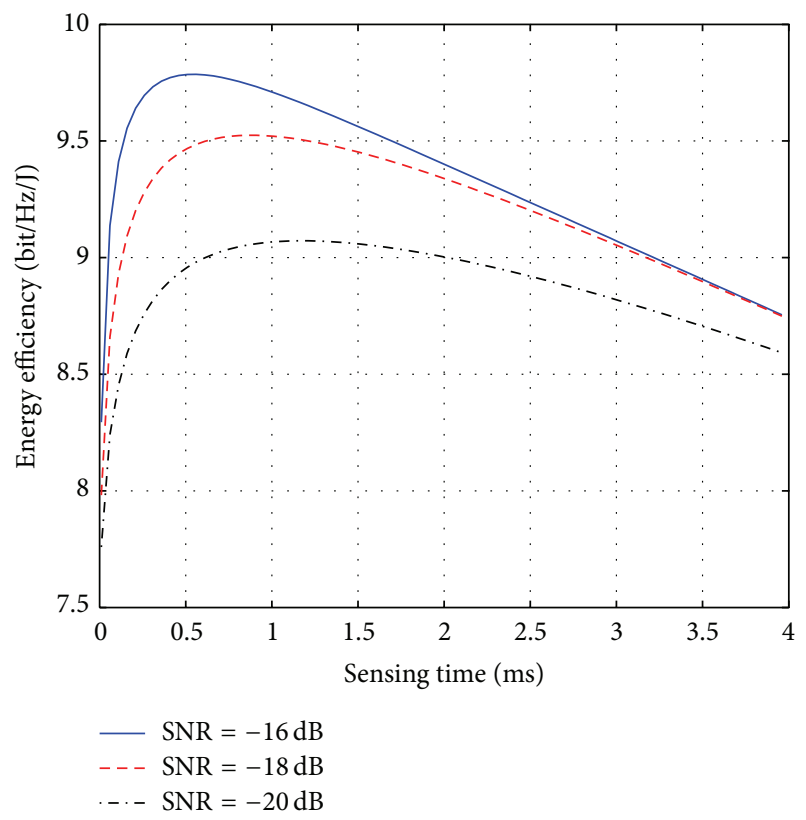

FIGURE 2: Energy efficiency versus sensing time.

In Figure 3, the energy efficiency versus the average interference power threshold is compared between different schemes. In the proposed scheme, the sensing time and power allocation are jointly optimized. In scheme I, the sensing time is optimized while all SUs share the same transmit power which satisfies the constraint (15f). And in scheme II, the power allocation is optimized with a fixed sensing time $\tau_{s}=5 \mathrm{~ms}$. It can be observed that the proposed scheme outperforms scheme I and scheme II. Energy efficiency in all schemes increases when the average interference power 


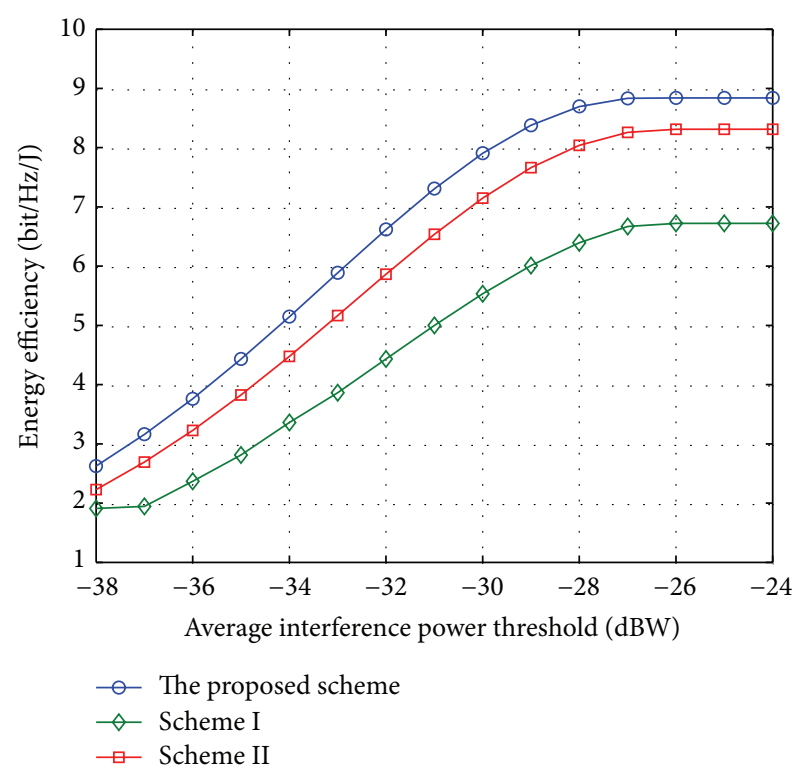

FIGURE 3: Energy efficiency versus average interference power threshold.

threshold initially increases. However, when the average interference power threshold is increased further, the energy efficiency does not increase any more as the constraint of the average interference power is loose.

\section{Conclusion}

In this paper, we consider the scenario that multiple SUs jointly sense one licensed spectrum band which can be divided into multiple subbands and each SU operates exclusively in one of the subbands when the PU is detected to be absent. We focus on the joint optimization between the spectrum sensing time and power allocation to maximize energy efficiency of the CRN, with limited interference to the PU. During the optimizing process, the target detection probability and the quality of service parameter have also been taken into consideration. An optimization algorithm combined with fractional programming to solve the optimization problem is proposed. Numerical results show that the performance improvement is achieved by joint optimization of sensing time and power allocation.

\section{Conflict of Interests}

The authors declare that there is no conflict of interests regarding the publication of this paper.

\section{Acknowledgments}

This work is supported by National Nature Science Foundation of China (nos. 11301071, 61372106, 61102068, 61172077, and 61223001), Key Special Project of National Science and Technology (no. 2013ZX03003006), National 863 High Technology Development Project (no. 2013AA013601), Research
Fund of National Mobile Communications Research Laboratory, Southeast University (no. 2013A04, 2014B01), and CPSF (2013M531244).

\section{References}

[1] A. Ghasemi and E. S. Sousa, "Spectrum sensing in cognitive radio networks: requirements, challenges and design tradeoffs," IEEE Communications Magazine, vol. 46, no. 4, pp. 32-39, 2008.

[2] J. Mitola III and G. Q. Maguire Jr., "Cognitive radio: making software radios more personal," IEEE Personal Communications, vol. 6, no. 4, pp. 13-18, 1999.

[3] L. Y. Li, X. W. Zhou, H. B. Xu, G. Y. Li, D. D. Wang, and A. Soong, "Energy-efficient transmission in cognitive radio networks," in Proceedings of the 7th IEEE Consumer Communications and Networking Conference (CCNC '10), pp. 1-5, Las Vegas, Nev, USA, January 2010.

[4] Z. Shi, K. C. Teh, and K. H. Li, "Energy-efficient joint design of sensing and transmission durations for protection of primary user in cognitive radio systems," IEEE Communications Letters, vol. 17, no. 3, pp. 465-468, 2013.

[5] E. C. Y. Peh, Y. C. Liang, Y. L. Guan, and Y. Y. Pei, "Energyefficient cooperative spectrum sensing in cognitive radio networks," in Proceedings of the IEEE Global Communications Conference, pp. 1-5, 2011.

[6] Y. Pei, Y. Liang, K. C. Teh, and K. H. Li, "Energy-efficient design of sequential channel sensing in cognitive radio networks: optimal sensing strategy, power allocation, and sensing order," IEEE Journal on Selected Areas in Communications, vol. 29, no. 8, pp. 1648-1659, 2011.

[7] K. Illanko, M. Naeem, A. Anpalagan, and D. Androutsos, "Low complexity energy efficient power allocation for green cognitive radio with rate constraints," in Proceedings of the IEEE Global Communications Conference (GLOBECOM '12), pp. 3377-3382, December 2012.

[8] B. Wang, K. J. R. Liu, and T. C. Clancy, "Evolutionary cooperative spectrum sensing game: how to collaborate?" IEEE Transactions on Communications, vol. 58, no. 3, pp. 890-900, 2010.

[9] R. Fan, H. Jiang, Q. Guo, and Z. Zhang, "Joint optimal cooperative sensing and resource allocation in multichannel cognitive radio networks," IEEE Transactions on Vehicular Technology, vol. 60, no. 2, pp. 722-729, 2011.

[10] I. F. Akyildiz, B. F. Lo, and R. Balakrishnan, "Cooperative spectrum sensing in cognitive radio networks: a survey," Physical Communication, vol. 4, no. 1, pp. 40-62, 2011.

[11] Y. Liang, Y. Zeng, E. C. Y. Peh, and A. T. Hoang, "Sensingthroughput tradeoff for cognitive radio networks," IEEE Transactions on Wireless Communications, vol. 7, no. 4, pp. 1326-1337, 2008.

[12] E. C. Y. Peh, Y. Liang, Y. L. Guan, and Y. Zeng, "Optimization of cooperative sensing in cognitive radio networks: a sensingthroughput tradeoff view," IEEE Transactions on Vehicular Technology, vol. 58, no. 9, pp. 5294-5299, 2009.

[13] S. Stotas and A. Nallanathan, "Optimal sensing time and power allocation in multiband cognitive radio networks," IEEE Transactions on Communications, vol. 59, no. 1, pp. 226-235, 2011.

[14] W. Dinkelbach, “On nonlinear fractional programming," Management Science, vol. 13, no. 7, pp. 492-498, 1967. 
[15] Y. Y. Pei, Y. C. Liang, K. C. Teh, and K. H. Li, "How much time is needed for wideband spectrum sensing?" IEEE Transactions on Wireless Communications, vol. 8, no. 11, pp. 5466-5471, 2009.

[16] S. Boyd and L. Vandenberghe, Convex Optimization, Cambridge University Press, Cambridge, UK, 2004. 


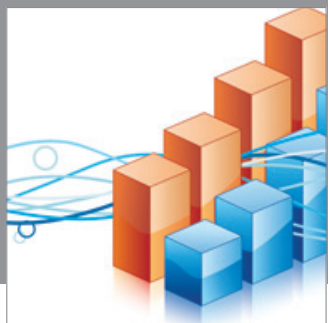

Advances in

Operations Research

mansans

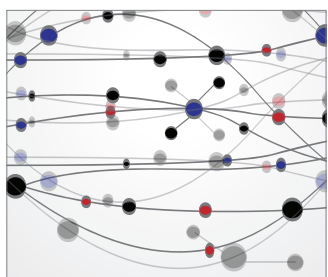

The Scientific World Journal
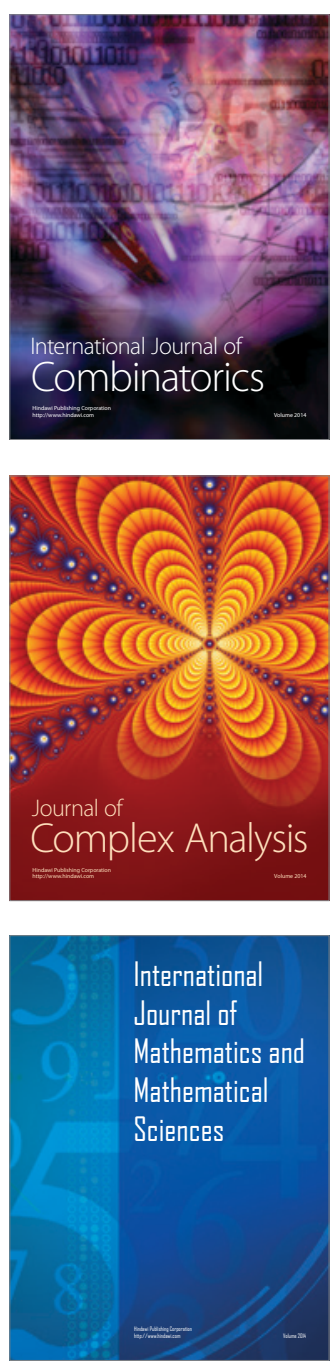
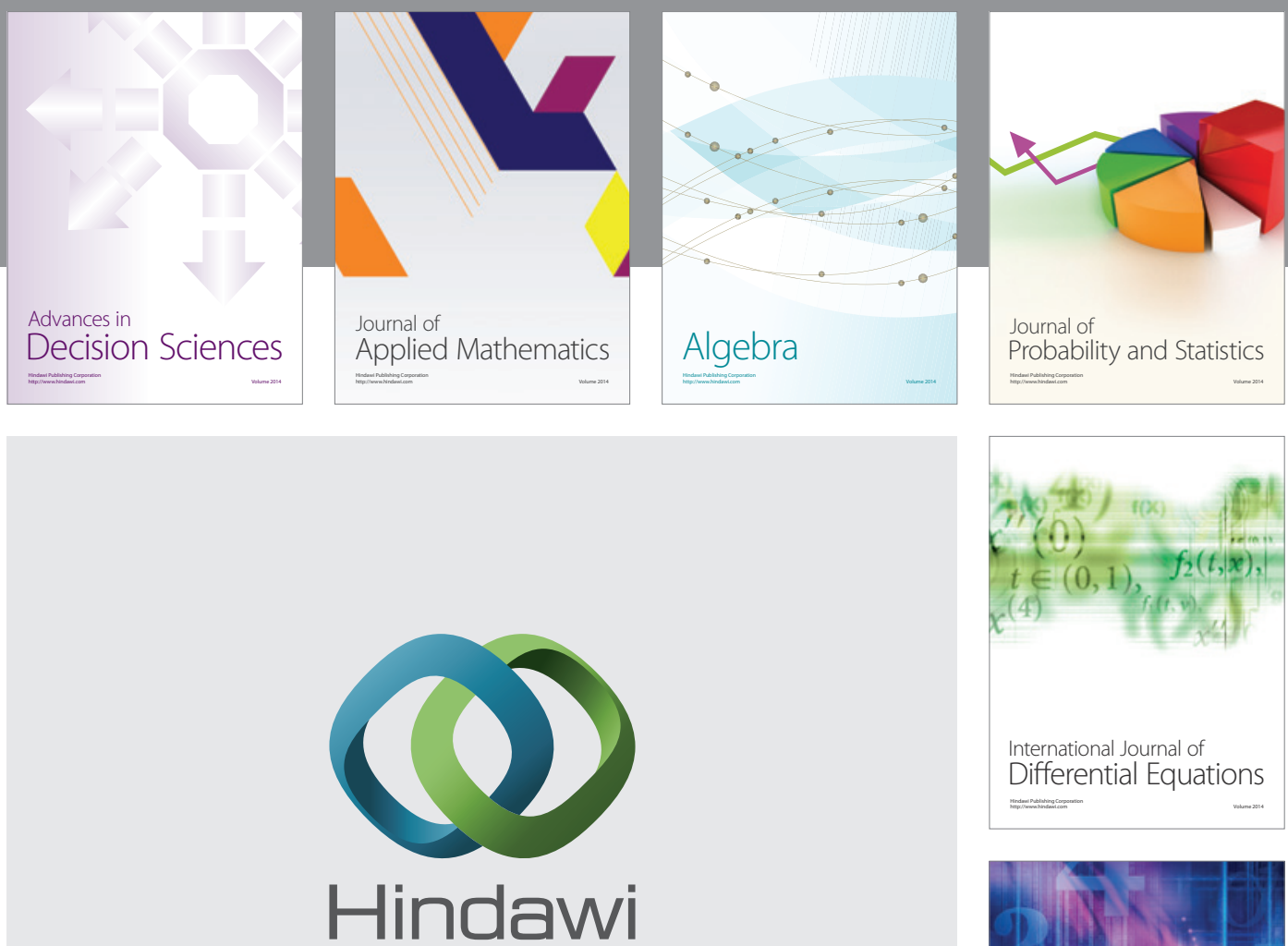

Submit your manuscripts at http://www.hindawi.com
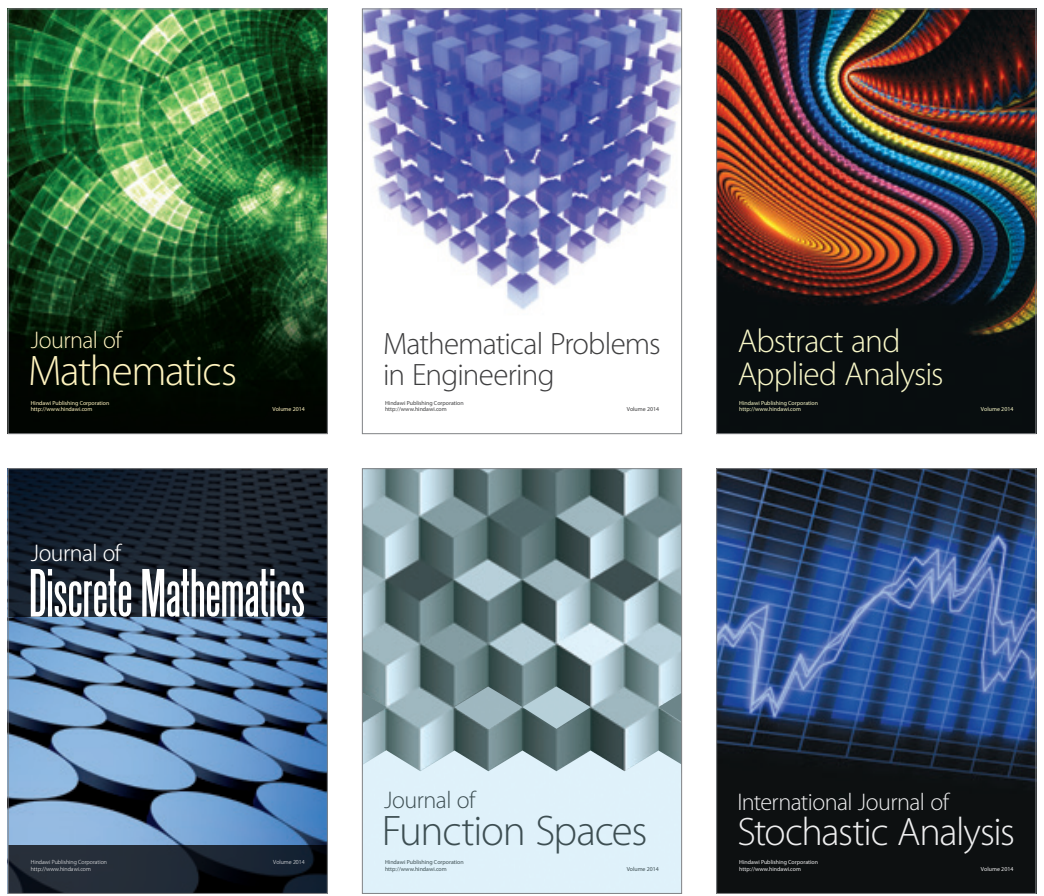

Journal of

Function Spaces

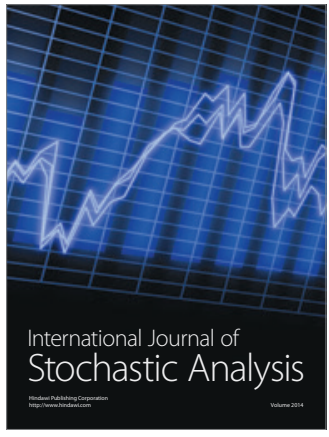

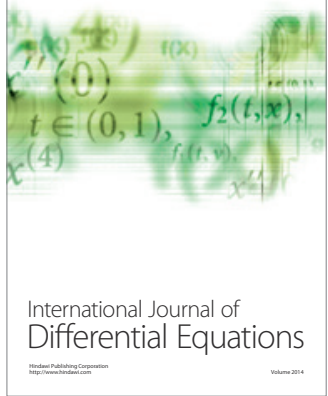
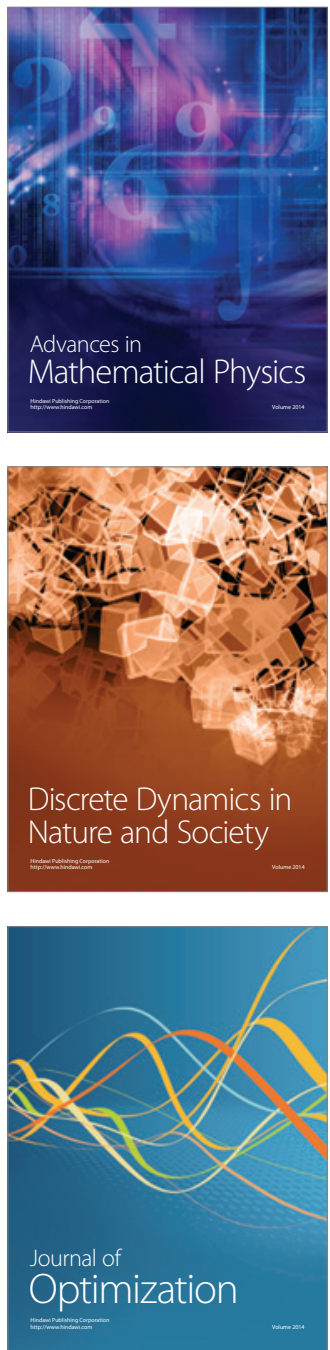